\title{
Indication of a size-dependent transition from molecular to dissociative chemisorption on clusters
}

\author{
S. Burkart, N. Blessing, and G. Ganteför \\ Fakultät für Physik, Universität Konstanz, 78457 Konstanz, Germany
}

\begin{abstract}
We report experimental indications for a size-dependent change of the chemical nature of chemisorption on small atomic clusters. We studied chemisorption of atomic hydrogen on negatively charged $\mathrm{Ti}_{n}{ }^{-}$clusters using mass and photoelectron spectroscopy. Our experimental data support the assumption that for clusters with up to four Ti atoms, adsorption of intact $\mathrm{H}_{2}$ molecules is the energetically preferred configuration For larger $\mathrm{Ti}_{n}$ clusters with $n>4$, dissociative hydrogen chemisorption is the most stable configuration forming clusters with an electronic structure similar to bulk $\mathrm{TiH}_{2}$.
\end{abstract}

Small particles (clusters) exhibit exceptional chemical properties, e.g., reaction rates of $\mathrm{H}_{2}$ with $\mathrm{Fe}_{n}$ (Ref. 1) or $\mathrm{Nb}_{n}$ (Refs. 2 and 3) clusters vary by several orders of magnitude depending on the number of metal atoms. The dramatic size dependence has been attributed to entrance channel barriers created by special geometries ${ }^{4}$ or to the exceptional electronic structure ${ }^{5}$ of the small metal particles. For the reaction $\mathrm{Fe}_{n}+\mathrm{H}_{2}$, the correlation between rates and ionization potentials (IP's) has been explained by a charge-transfer model.,

For the reaction of $\mathrm{N}_{2}$ with $\mathrm{W}_{n}$ clusters, two different reaction channels, molecular and dissociative chemisorption, compete with each other. ${ }^{7,8}$ The reaction rates of the dissociative channel vary by several orders of magnitude with cluster size, and no dissociative chemisorption has been observed for small clusters with $n<15$. For all $\mathrm{W}_{n}$ clusters the dissociatively bound $\mathrm{N}_{2}$ is thought to be the most stable configuration but for $n<15$ the barriers are too high, and adsorption takes place in the metastable precursor state of molecular bound $\mathrm{N}_{2}$. Analogous to the case of $\mathrm{W}$ surfaces, these observations are explained by geometric effects, i.e., the availability of particular adsorption sites. ${ }^{7,8}$

In all these cases the nature of the ground-state configu ration of the chemisorption bond (molecular or dissociative) is believed to be the same for clusters and corresponding surfaces, independent of cluster size. ${ }^{1-8}$ Of particular note, so far there are no experimental indications for molecular chemisorption of $\mathrm{H}_{2}$ to any metal cluster or surface. $\mathrm{H}_{2}$ always seems to dissociate.

In this Brief Report we address the question of whether the chemical nature of chemisorption might depend fundamentally on cluster size independent of the existence of energy barriers. Such a fundamental dependence on cluster size should occur according to a simple model of CO chemisorption on transition metal surfaces. ${ }^{9} \mathrm{CO}$ is bound as a molecule to $\mathrm{Cu}, \mathrm{Ni}$, or Pd surfaces, while it dissociates on surfaces of the "'early" transition metals (i.e., the ones on the left side of the periodic table) like Sc or Ti. This effect is explained by the differences in the work functions between the "early", and the "late" transition metals. For a low work function (Sc,Ti) the $d$ electrons can be removed more easily and charge donation from the metal $d$ band into the antibonding $2 \pi^{*}$ orbital of CO is increased. The bond is broken and CO is bound dissociatively. For a higher work function there is insufficien charge transfer and CO binds molecularly.
The IP's of clusters exhibit a pronounced size dependence. ${ }^{10}$ The IP's of the atom and small clusters are considerably higher than the corresponding bulk work functions and, in accordance with the picture described above, molecular chemisorption should be the preferred type of bonding. For small metal clusters there might even be a chance for molecular chemisorption of $\mathrm{H}_{2}$ to be the most stable state despite the strong tendency of $\mathrm{H}_{2}$ to dissociate.

This question cannot be solved in reaction rate experiments with molecular adsorbates such as $\mathrm{H}_{2}$ or $\mathrm{N}_{2}$, because it is not possible in principle to distinguish between a high barrier for dissociative chemisorption and the nonexistence of this type of adsorption. It can be studied using atomic gases such as $\mathrm{H}$, which probably react without barriers. In this case, dissociative and molecular chemisorption can easily be distinguished by mass spectroscopy: molecular chemisorption yields even numbers of adsorbed $\mathrm{H}$ atoms only, while dissociative chemisorption results in an uptake of even and odd numbers of $\mathrm{H}$ atoms.

Here we focus on the chemisorption of hydrogen on small $\mathrm{Ti}$ clusters. $\mathrm{H}_{2}$ chemisorption on $\mathrm{Ti}$ bulk surfaces is dissociative. ${ }^{11}$ For a single $\mathrm{Ti}$ atom, $\mathrm{H}_{2}$ chemisorption should be nondissociative because of energetic considerations: the binding energy of the TiH diatomic molecule is $1.96 \mathrm{eV},{ }^{12}$ while the $\mathrm{H}_{2}$ molecule is bound by $4.48 \mathrm{eV} .{ }^{13}$ As long as the binding energy of $\mathrm{H}_{2}$ is larger than twice that of a single $\mathrm{Ti}-\mathrm{H}$ bond, chemisorption will be nondissociative. This analysis is supported by the observation of molecularly bound $\mathrm{Ti}^{+}\left(\mathrm{H}_{2}\right)_{1-6}$ clusters. ${ }^{14}$ In contrast, a single hydrogen atom is bound by $\approx 3.0 \mathrm{eV}$ to a Ti surface, so for bulk surfaces dissociative chemisorption is the lower energy channel with a total gain in binding energy of $2 \times 3.0 \mathrm{eV}-4.48 \mathrm{eV}$ $\approx 1.5 \mathrm{eV} .{ }^{15}$ Accordingly, a size-dependent transition from molecular to dissociative $\mathrm{H}$ chemisorption must occur for $\mathrm{Ti}_{n}$ clusters.

We produced $\mathrm{Ti}_{n} \mathrm{H}_{m}{ }^{-}$cluster anions by the reaction of $\mathrm{Ti}_{n}{ }^{-}$clusters with atomic hydrogen. For small clusters only even numbers of chemisorbed hydrogen atoms were found, while for larger $\mathrm{Ti}_{n} \mathrm{H}_{m}{ }^{-}$clusters, with $n>4$, even and odd values of $m$ were observed. In addition, we have studied the electronic structure of size-selected bare and reacted $\mathrm{Ti}_{n} \mathrm{H}_{m}{ }^{-}$ cluster anions by photoelectron spectroscopy and have found a dramatic change of the observed spectroscopic pattern occurring at $n=4$. These changes in both mass and photoelec- 
(a)

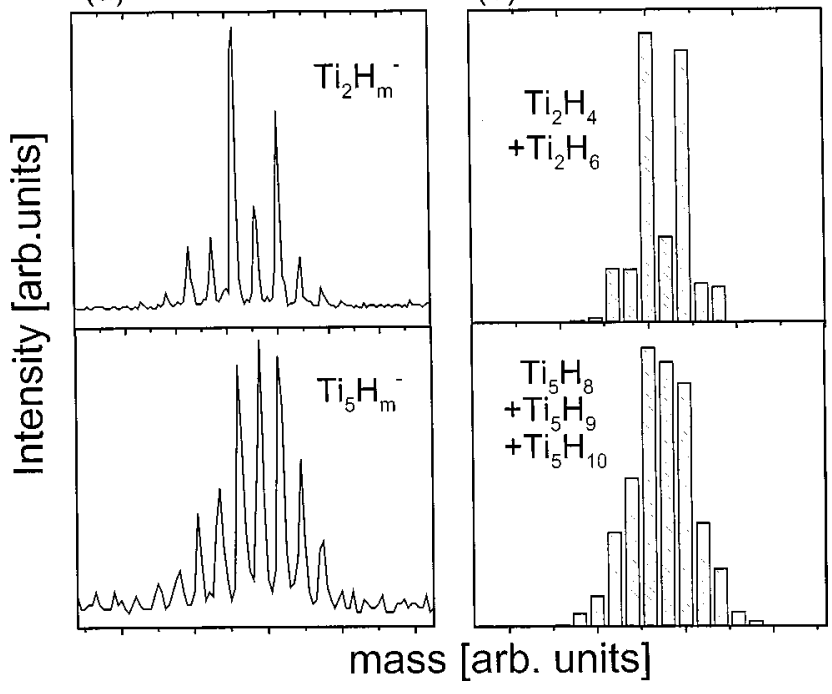

FIG. 1. (a) Two features observed in the mass spectrum of reacted $\mathrm{Ti}_{n} \mathrm{H}_{m}{ }^{-}$clusters. The two groups of peaks are assigned to $\mathrm{Ti}_{2} \mathrm{H}_{m}{ }^{-}(m=4,6)$ and $\mathrm{Ti}_{5} \mathrm{H}_{m}{ }^{-}(m=8,9,10)$. Each cluster with a specifie $n$ and $m$ gives rise to a group of peaks with one intense center line corresponding to the isotope distribution of the Ti atom. Each of the two features is a superposition of such isotope patterns from clusters with different $m$. (b) Simulations of the experimental data shown in (a). The intensity distribution for $\mathrm{Ti}_{2} \mathrm{H}_{m}{ }^{-}$can be fitte by a superposition of $\mathrm{Ti}_{2} \mathrm{H}_{4}{ }^{-}+\mathrm{Ti}_{2} \mathrm{H}_{6}{ }^{-}$(relative intensities $1: 1)$. In general, the observation of intense peaks separated by 2 amu indicates an uptake of intact $\mathrm{H}_{2}$ molecules only. The pattern observed for $\mathrm{Ti}_{5} \mathrm{H}_{m}{ }^{-}$is reproduced by adding the isotope distributions of $\mathrm{Ti}_{5} \mathrm{H}_{8}{ }^{-}+\mathrm{Ti}_{5} \mathrm{H}_{9}{ }^{-}+\mathrm{Ti}_{5} \mathrm{H}_{10}{ }^{-}$(relative intensities 1:1:1).

tron spectra may indicate a transition from molecular to dissociative hydrogen chemisorption.

The experimental setup consists of a cluster ion source, a time-of-fligh mass spectrometer, and an electron time-offligh spectrometer. ${ }^{16}$ Titanium cluster anions are produced using a pulsed arc cluster ion source (PACIS). Reacted clusters are generated by mixing $\mathrm{H}_{2}$ with $\mathrm{He}$ carrier gas and dissociating it with an electric arc. The bare and reacted cluster anions are cooled down to approximately room temperature. The cluster anions are mass-separated in a reflectio time-of-fligh mass spectrometer with a resolution of $m / \Delta m=400$. Photoelectron spectra are recorded using a "magnetic bottle" time-of-fligh electron spectrometer operated at low energy resolution $(\approx 50 \mathrm{meV})$ due to the rather weak electron signal from the reacted clusters. The spectra of the $\mathrm{Ti}_{n} \mathrm{H}_{m}{ }^{-}$clusters are recorded using a Nd:YAG laser (YAG denotes yttrium aluminum garnet) with a photon energy of $3.49 \mathrm{eV}$ and an intensity of $\approx 10 \mathrm{~mJ} / \mathrm{cm}^{2}$. The electron spectrometer is calibrated using known atomic transitions of $\mathrm{Cu}_{1}{ }^{-1}$ and $\mathrm{Au}_{1}{ }^{-}$. Spectra of the bare $\mathrm{Ti}_{n}{ }^{-}$clusters are in excellent agreement with data from $\mathrm{Wu}$, Desai, and Wang. ${ }^{17}$

In the mass spectra, features assigned to a particular cluster consist of several narrow peaks reflectin the isotope distribution of Ti. Each $\mathrm{Ti}_{n} \mathrm{H}_{m}{ }^{-}$cluster gives rise to a single intense peak accompanied by two weaker lines on both sides. As an example, Fig. 1(a) displays two features in the mass spectrum of the $\mathrm{Ti}_{n} \mathrm{H}_{m}{ }^{-}$clusters, which are assigned to $\mathrm{Ti}_{2} \mathrm{H}_{m}{ }^{-}$and $\mathrm{Ti}_{5} \mathrm{H}_{m}{ }^{-}$. The intensity distribution of the feature

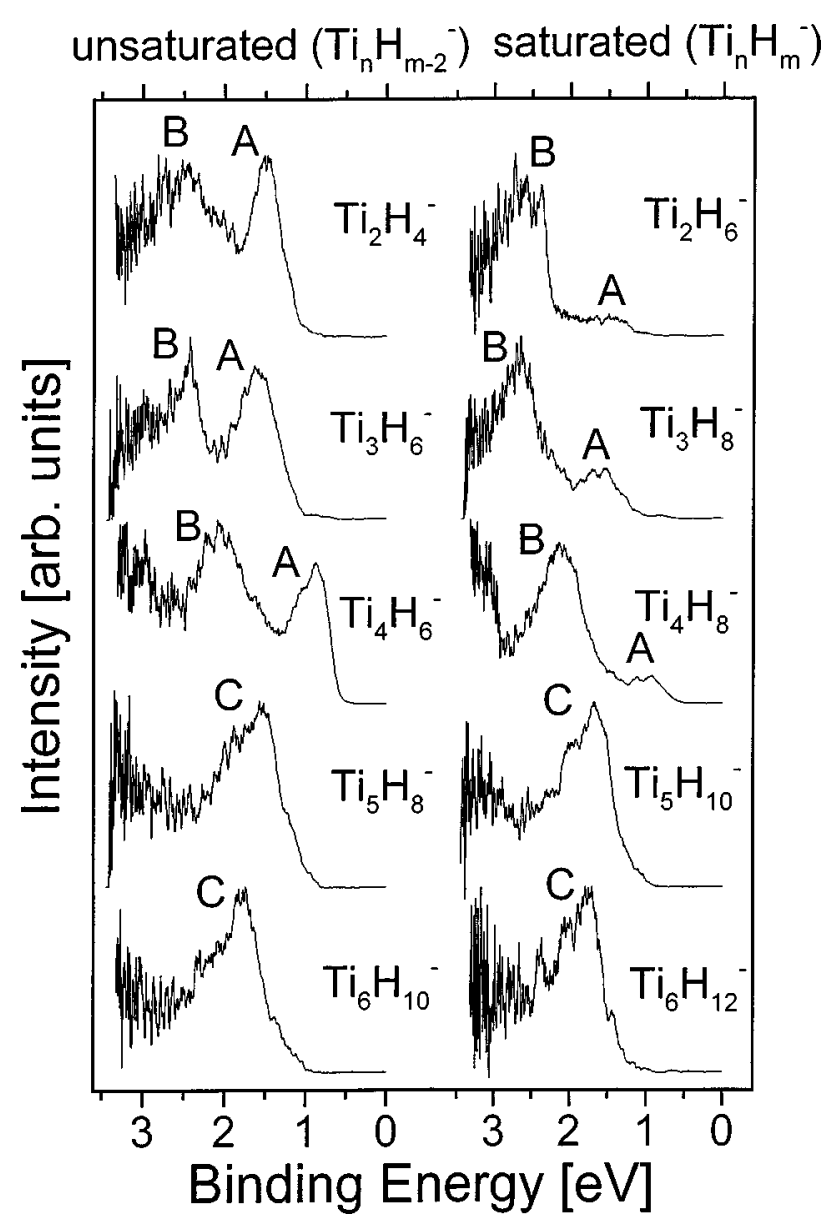

FIG. 2. Photoelectron spectra of $\mathrm{Ti}_{n} \mathrm{H}_{m}{ }^{-}$clusters recorded with a photon energy of $3.49 \mathrm{eV}$. The spectra of small clusters with $n$ $\leqslant 4$ exhibit two peaks $(A, B)$ with a strong decrease of intensity of peak $A$ for the saturated species $\left(\mathrm{Ti}_{2} \mathrm{H}_{6}{ }^{-}, \mathrm{Ti}_{3} \mathrm{H}_{8}{ }^{-}, \mathrm{Ti}_{4} \mathrm{H}_{8}{ }^{-}\right)$. For larger clusters only a single feature $(C)$ is observed.

assigned to $\mathrm{Ti}_{2} \mathrm{H}_{m}$ - is a superposition of contributions from $\mathrm{Ti}_{2} \mathrm{H}_{4}{ }^{-}$and $\mathrm{Ti}_{2} \mathrm{H}_{6}{ }^{-}$only [Fig. 1(b)]. That of $\mathrm{Ti}_{5} \mathrm{H}_{m}{ }^{-}$is a sum of contributions from $\mathrm{Ti}_{5} \mathrm{H}_{8}^{-}, \mathrm{Ti}_{5} \mathrm{H}_{9}{ }^{-}$, and $\mathrm{Ti}_{5} \mathrm{H}_{10}{ }^{-}$[Fig. 1(b)]. A pattern similar to that of $\mathrm{Ti}_{2} \mathrm{H}_{m}{ }^{-}$indicates an uptake of intact $\mathrm{H}_{2}$ molecules only and is observed for all smaller clusters with $n \leqslant 4$. All larger clusters show no preference for chemisorption of even $m$, as it would be expected for dissociative chemisorption.

No signal could be detected for $\mathrm{Ti}_{1} \mathrm{H}_{m}{ }^{-}$, which can be explained by the low electron affinit of the Ti atom. It was not possible to adjust the $\mathrm{H}_{2}$ load in the source to such low values that $\mathrm{Ti}_{n} \mathrm{H}_{m}{ }^{-}$clusters with a lower hydrogen uptake could be generated at sufficien intensity. Only saturated clusters and species with one or two $\mathrm{H}$ atoms less than the saturation limit are produced efficiently

Figure 2 displays photoelectron spectra of reacted $\mathrm{Ti}_{m} \mathrm{H}_{m}{ }^{-}$clusters with $n=2-6$. The spectra of clusters with up to four Ti atoms exhibit two peaks $(A, B)$ separated by about $1 \mathrm{eV}$. For the saturated species $\left(\mathrm{Ti}_{2} \mathrm{H}_{6}{ }^{-}, \mathrm{Ti}_{3} \mathrm{H}_{8}{ }^{-}\right.$, $\mathrm{Ti}_{4} \mathrm{H}_{8}{ }^{-}$), peak $a$ is diminished. Due to the isotope distribution there is about a $20 \%$ contribution from unsaturated clusters (assuming similar photodetachment cross sections), which is responsible for the residual intensity of peak $A$. Therefore, within the accuracy of the experiment, hydrogen saturation results in the disappearance of peak $A$ for $n=2,3$, and 4. 
For larger $\mathrm{Ti}_{n} \mathrm{H}_{m}{ }^{-}$clusters, with $n>4$, only a single peak $C$ is visible. We also recorded spectra of some selected larger clusters (not shown) and found a similar pattern only with a single peak and no indication of a change of the electronic structure due to saturation: i.e., the pattern observed for $\mathrm{Ti}_{5} \mathrm{H}_{m}{ }^{-}$and $\mathrm{Ti}_{6} \mathrm{H}_{m}{ }^{-}$extends to larger cluster sizes. The spectra of $\mathrm{Ti}_{5} \mathrm{H}_{9}{ }^{-}$and $\mathrm{Ti}_{6} \mathrm{H}_{11}{ }^{-}$(not shown) are similar to those shown with even $m$.

We assign the single feature $C$ to the formation of $\left(\mathrm{TiH}_{2}\right)_{n}{ }^{-}$clusters, which can be viewed as fragments of bulk $\mathrm{TiH}_{2}$. This explains the observed saturation limits of $\mathrm{Ti}_{5} \mathrm{H}_{10}{ }^{-}$ and $\mathrm{Ti}_{6} \mathrm{H}_{12}{ }^{-}$. The assignment is supported by the observation of a single peak close to the Fermi level $\left(E_{F}\right)$ in the valence-band photoemission spectrum of bulk $\mathrm{TiH}_{2} .{ }^{18}$ This feature is similar in shape and position to peak $C$ in Fig. 2. In $\mathrm{TiH}_{2}$ a covalent bond is formed between the hydrogen $1 s$ orbital and some metal $3 d$ and $4 s / p$ orbitals. The metal $3 d$ density of states is modifie and the photoemission spectrum in the binding energy (BE) range of the metal $3 d$ orbitals is dominated by a single peak. ${ }^{18}$ In the case of an unsaturated cluster with one or two missing $\mathrm{H}$ atoms, the number of electrons occupying these metal $3 d$-derived orbitals is increased by one or two, but the overall structure of the valence band might remain almost unchanged. This explains why saturation does not affect the pattern observed in the photoemission spectra of $\mathrm{Ti}_{5} \mathrm{H}_{m}{ }^{-}$and $\mathrm{Ti}_{6} \mathrm{H}_{m}{ }^{-}$. In bulk $\mathrm{TiH}_{2}$ the hydrogen is dissociated and we conclude that in clusters with $n>4$ hydrogen is bound dissociatively in agreement with the mass spectra.

The different pattern observed for the smaller $\mathrm{Ti}_{n} \mathrm{H}_{m}{ }^{-}$ clusters can be explained by molecular chemisorption. In this case, the bond may be formed by charge donation from the metal $3 d$ orbitals into the antibonding $\sigma^{*}$ orbital of $\mathrm{H}_{2}$ analogous to the "backdonation" mechanism of $\mathrm{CO}$ chemisorption. ${ }^{19}$ The newly formed $3 d / \sigma^{*}$ hybrid orbital accommodates two electrons from the metal and, for the neutral cluster, saturation corresponds to the depletion of nonbonding electrons from the metal $3 d$ orbitals. This crude picture gives a possible explanation for the disappearance of peak $A$ in Fig. $2 .^{20}$ For example, $\mathrm{Ti}_{2}$ has eight valence electrons with two electrons in a $4 s$-derived $\sigma$ orbital forming the

${ }^{1}$ R. L. Whetten, D. M. Cox, D. J. Trevor, and A. Kaldor, Phys. Rev. Lett. 54, 1494 (1985).

${ }^{2}$ J. L. Elkind, F. D. Weiss, J. M. Alford, R. T. Laaksonen, and R. E. Smalley, J. Chem. Phys. 88, 5215 (1988).

${ }^{3}$ M. R. Zakin, R. O. Brickman, D. M. Cox, and A. Kaldor, J. Chem. Phys. 88, 3555 (1988).

${ }^{4}$ A. Berces, P. A. Hackett, Li Lan, S. A. Mitchell, and D. M. Rayner, J. Chem. Phys. 108, 5476 (1998).

${ }^{5}$ H. Kietzmann, J. Morenzin, P. S. Bechthold, G. Ganteför, and W. Eberhardt, J. Chem. Phys. 109, 2275 (1998).

${ }^{6}$ J. Conceicao, R. T. Laaksonen, L.-S. Wang, T. Guo, P. Nordlander, and R. F. Smalley, Phys. Rev. B 51, 4668 (1995).

${ }^{7}$ S. A. Mitchell, D. M. Rayner, T. Bartlett, and P. A. Hackett, J. Chem. Phys. 1104, 4012 (1996).

${ }^{8}$ L. Holmgren, M. Andersson, and A. Rosén, J. Chem. Phys. 109, 3232 (1998). bond between the two metal atoms. The remaining six electrons give rise to the chemisorption of three $\mathrm{H}_{2}$ molecules. In larger clusters, up to $n=4$, a larger number of electrons is consumed for metal-metal bonds, reducing the saturation limit.

The preference for molecular chemisorption in the case of small $\mathrm{Ti}_{n}$ clusters can be explained by reduced charge transfer $^{21}$ due to the higher binding energy of the metal $3 d$ orbitals. ${ }^{9}$ The IP's of small clusters, which give a rough estimate of the binding energies of the uppermost occupied $3 d$ orbitals, are significantl higher than the bulk work function. ${ }^{10}$ This is caused by reduced screening of a $3 d$ hole in a small cluster and is a result of the lower number of nearest neighbors in clusters.

In conclusion, we have observed a change of the spectroscopic pattern in both the mass and the photoelectron spectra of $\mathrm{Ti}_{n} \mathrm{H}_{m}{ }^{-}$clusters produced by reactions with atomic hydrogen. For small clusters with $n \leqslant 4$ only even $m$ are observed, indicating molecular $\mathrm{H}_{2}$ adsorption, while for larger clusters even and odd values of $m$ are detected with uniform abundances. The corresponding photoelectron spectra indicate a change of electronic structure between $n=4$ and $n=5$. These experimental finding might be a firs hint for a fundamental transition in the nature of the chemisorption bond: in small clusters hydrogen might be bound molecularly and in clusters with more than four Ti atoms it is bound dissociatively. The driving force of such a transition is the change of the binding energy of the $3 d$ orbitals, which decreases with increasing cluster size. Because atomic hydrogen has been used for the reaction, effects of barriers can be mostly excluded and the transition reflect a fundamental change of the ground-state configuration This effect is probably not restricted to the system $\mathrm{Ti}_{n}+\mathrm{H}_{2}$ and should take place in many other systems. It gives some insight into the reasons for the exceptional catalytic properties of small particles and for the independence of these properties on the exact geometric structures of the particles.

We thank W. E. Evenson, E. Recknagel, D. Kreisle, and W. Eberhardt for their support and fruitful discussions. The additional support of the Deutsche Forschungsgemeinschaft is acknowledged.

${ }^{9}$ R. Hoffmann, Rev. Mod. Phys. 60, 601 (1988) (see diagram 38 on p. 623).

${ }^{10}$ K.-H. Meiwes-Broer, Hyperfin Interact. 89, 263 (1994).

${ }^{11}$ P. Cremaschi and J. L. Whitten, Phys. Rev. Lett. 46, 1242 (1981).

${ }^{12}$ Organometallic Ion Chemistry, edited by Ben Freiser (Kluwer Academic, Dordrecht, 1996).

${ }^{13}$ K. P. Huber and G. Herzberg, Molecular Spectra and Molecular Structure: IV. Constants of Diatomic Molecules (Van Nostrand Reinhold, New York, 1979).

${ }^{14}$ J. E. Bushnell, P. Maitre, P. R. Kemper, and M. T. Bowers, J. Chem. Phys. 106, 10153 (1997).

${ }^{15}$ P. Nordlander, S. Holloway, and J. K. Norskov, Surf. Sci. 136, 59 (1984).

${ }^{16}$ Chia-Yen Cha, G. Ganteför, and W. Eberhardt, Rev. Sci. Instrum. 63, 5661 (1992).

${ }^{17}$ Hongbin Wu, S. R. Desai, and Lai-Sheng Wang, Phys. Rev. Lett. 
76, 212 (1996).

${ }^{18}$ D. E. Eastman, Solid State Commun. 10, 933 (1972).

${ }^{19}$ G. Schulze Icking-Konert, H. Handschuh, G. Ganteför, and W. Eberhardt, Phys. Rev. Lett. 76, 1047 (1996), and references therein.

${ }^{20}$ Since there is one additional electron in the anion, the disappearance of peak $A$ needs more consideration. The additional electron probably occupies a $3 d$-derived orbital. With each chemisorbed $\mathrm{H}_{2}$ molecule, two $3 d$ electrons are transferred into the new $\sigma^{*} / 3 d$ hybrid orbital and the remaining $3 d$ electrons can shift to more stable $3 d$ orbitals. Therefore, peaks $A$ and $B$ are assigned to emission from metal $3 d$ orbitals and the disappearance of peak $A$ corresponds to the transfer of the last two electrons in to the $\sigma^{*} / 3 d$ hybrid orbital. Peak $B$ is assigned to emission from the most stable $3 d$ metal state, which accommodates the last remaining $3 d$ electron (the additional one of the anion) in case of saturation.

${ }^{21}$ In the anion the additional electron will probably increase the charge transfer into the antibonding $\sigma^{*}$ orbital. However, it seems unlikely that the additional charge is localized at a single $\mathrm{H}_{2}$ ligand. More likely, it is delocalized and distributed over the ligands, reducing its effect on charge transfer, analogous to the case of $\mathrm{Pt}_{3}\left(\mathrm{CO}_{6}\right)_{6}{ }^{-}$(Ref. 19). 